\title{
Review Article \\ The Role of Metal Regulatory Proteins in Brain Oxidative Stress: A Tutorial
}

\author{
Wayne Briner \\ Department of Psychology, University of Nebraska at Kearney, Kearney, NE 68849, USA \\ Correspondence should be addressed to Wayne Briner, brinerw@unk.edu
}

Received 14 September 2012; Revised 5 November 2012; Accepted 8 November 2012

Academic Editor: Cinzia Signorini

Copyright () 2012 Wayne Briner. This is an open access article distributed under the Creative Commons Attribution License, which permits unrestricted use, distribution, and reproduction in any medium, provided the original work is properly cited.

The proteins that regulate the metabolism of a metal must also play a role in regulating the redox activity of the metal. Metals are intrinsic to a substantial number of biological processes and the proteins that regulate those activities are also considerable in number. The role these proteins play in a wide range of physiological processes involves them directly and indirectly in a variety of disease processes. Similarly, it may be therapeutically advantageous to pharmacologically alter the activity of these metal containing proteins to influence disease processes. This paper will introduce the reader to a number of important proteins in both metal metabolism and oxidative stress, with an emphasis on the brain. Potential pharmacological targets will be considered.

\section{Introduction}

In many respects, metals have an undeserved bad name. They have been implicated in any number of disease processes (e.g., lead poisoning, mercury poisoning, and Wilson's disease). However, metals do not simply enter the brain as ions and begin wreaking havoc via oxidative stress and other mechanisms. A variety of proteins are involved in the regulation of metal metabolism and the oxidative response. Many of these proteins play a primary role in this activity, in that regulating a particular metal seems to be the primary purpose of that protein. Other proteins, however, seem to play a secondary or more coincidental role. The number of proteins involved is substantial, and many are involved in iron or copper metabolism due to the redox activity of those metals. This paper will provide a survey of a number of the proteins involved in metal induced oxidative stress, with an emphasis on the brain. It is certainly not an exhaustive list, nor an exhaustive review. Rather, it is intended to give those interested in metal metabolism, but where it may not be their primary research interest, some background in the area. While the brain is the organ of focus, other organs may be mentioned to provide insight into the function of a molecule, but will not be the primary target of discussion. Because this is meant as a tutorial and not an exhaustive review, references are provided at the end of each section to allow the reader to pursue a more in-depth pursuit of the topic as they so desire.

\section{Albumin}

Albumin is the protein found in the largest quantity in blood and is most well known for its role in regulating the osmotic pressure of blood. Albumin also contains a variety of binding sites for drugs, fatty acids, bilirubin, and metals. The degree to which substances bind with albumin depends on a variety of chemical characteristics including charge, size, and the solubility of the compound in question as well as the redox state of the albumin molecule. Albumin is synthesized by the liver and the impaired secretion of albumin has a significant impact on health. Additionally, disease states that effect the redox state of albumin can have a significant impact on health; renal failure and diabetes are particularly noteworthy in this regard, altering the metal binding capacity of albumin. A variety of binding sites are available on the albumin with the molecule consisting of three large domains and one small domain, and two subdomains are found on each of the larger domains. The molecule also contains 35 cysteine residues most of which are involved in intramolecular disulphide bonds but the one remaining cysteine can act as potential metal binding site. 
A variety of metals are bound by albumin, these include aluminum, cadmium, calcium, cobalt, copper, magnesium, manganese, mercury, nickel, potassium, sodium, and zinc. Binding for these metals can occur at the amino terminus of albumin or at a free sulphydryl group.

Because of its size and ubiquitous nature, albumin is a target for reactive oxygen species and may be an important systemic buffer for oxidative stress. While not typically glycosylated albumin can be glycated from prolonged exposure of glucose. This may occur in diabetes, where the percentage of glycated albumin can reach $30 \%$. Glycation of albumin can interfere with its ability to bind metals and other substances.

When oxidized or glycated, the structure of albumin changes typically leading to poorer binding of most substances. Poorer binding of metals is likely the result of these structural changes as illustrated by the release of cobalt from albumin during the ischemic and reperfusion changes seen during myocardial infarction. Indeed the release of cobalt from albumin stores is a marker for myocardial infarction. A decrease in metal binding means of course more free metal available to produce oxidative stress and other physiological effects.

Pharmacologic manipulation of albumin to improve the response to oxidative stress would be difficult. Insuring the supply of other circulating scavengers would probably be the most effective method of insuring that albumin performs its physiological role. Tailoring a compound to protect albumin from oxidative stress might be possible, but might alter the function of albumin in an undesirable manner. For more information see Oettl and Stauber 2007 [1] and Turell et al. 2009 [2].

\section{Amyloid Precursor Protein}

Amyloid precursor protein (APP) has a considerable name recognition for the role it plays in Alzheimer's disease (AD) and other forms of dementia. However, the role APP plays in dementia is due to errors in protein cleavage and folding and not an inherent property of APP. Indeed APP plays a number of vital roles in normal neuronal function and a variety of metals influence its activity.

APP and APP-like proteins are found throughout the animal kingdom and the structure is fairly well conserved. In humans, the APP gene is found on the long arm of chromosome 21 and, after being processed by 3 secretases, ranges from 365 to 770 amino acids in length. Abnormal cleavage by the secretases, in the presence of lipid rafts, may lead to the accumulation of amyloid seen in AD.

APP plays a role in a variety of tissues (e.g., connective and embryonic) but is most well studied in the brain. Its transmembrane structure allows it to play an important role in cell and synaptic adhesion and may guide neuronal growth. APP is upregulated during synaptogenesis, such as that seen during development and repair of neural tissue. The function of APP may extend to ordinary synaptic formation such as that seen during memory formation. However, the exact function that APP plays in these roles depends on the exact form of APP with APP-alpha playing a prosynaptic role and APP-beta playing a role in synaptic pruning, and even neural apoptosis.

APP also plays a role in metal metabolism, in particular copper, iron, and zinc. APP may play a crucial role in $\mathrm{Cu}$ efflux from the cell. Evidence for this comes from the presence of a Cu binding site on APP and that APP knockout mice show elevated intracellular $\mathrm{Cu}$ levels. Overexpression of APP leads to lowered CU levels in the cell. The role of APP in Fe regulation is suggested by the presence of a binding site and that overexpression of APP reduces intracellular Fe levels. Similar evidence indicates that APP plays a role in the removal of $\mathrm{Zn}$ from the intracellular environment.

Several lines of evidence suggest that disruption of metal metabolism, particularly of $\mathrm{Cu}$ and $\mathrm{Fe}$, play an important role in amyloid-beta ( $\mathrm{Ab}$ ) formation. $\mathrm{Cu}$ may play a direct role in $\mathrm{Ab}$ formation and accumulate in amyloid plaques, yet the neural tissues may suffer from an intracellular deficit of $\mathrm{Cu}$. Additionally, $\mathrm{Fe}$ and $\mathrm{Cu}$ may play a role in the formation of ROS adding to neural stress, which may be further exacerbated by the divergence of APP to the formation of amyloid plaques. Zn may also play a role by disrupting APPs role in $\mathrm{Fe}$ metabolism, but it is unclear if this is primary or secondary to the generation of $\mathrm{AD}$. In any event, metals clearly play a role in $\mathrm{AD}$, but the exact nature of that role is unclear.

In terms of pharmacological targets the metal binding site of APP holds promise as a means to manipulate the activity of various metals and the role they may subsequently play in the generation of AD. Some researchers have explored the use of chelators to alter the progress of $\mathrm{AD}$. The $\mathrm{Cu} / \mathrm{Zn}$ chelator clioquinol has shown some promising positive results on markers of AD. Supplementation with a variety of antioxidants has also been attempted, with very mixed results on $\mathrm{AD}$ markers. A more promising approach may be one that does not involve the use of metal, but rather inhibition of the BACE1 enzyme responsible for Ab formation. Several laboratories are examining lithium as a potential agent to alter APP metabolism. For further reading consult Finefrock et al., 2003 [3] and Maynard et al., 2005 [4] and Zheng and Koo, 2011 [5].

\section{Atx-1/Atox1/Copper Chaperone Peptides}

Atox 1 is a copper transport protein, or copper chaperone. It moves copper from one intracellular location to another, typically to a copper-ATPase. Because it binds free copper, it does exert antioxidant effects. This protein may alter the activity of other metal binding proteins, particularly those involved in $\mathrm{Zn}$ metabolism. Not all copper chaperones are equivalent; some are involved in transporting copper to the mitochondria, others to superoxide dismutases or copper ATPases. Relatively little exploration of how these peptides might be manipulated by drugs has been done. If some methods could be devised to increase the synthesis of these substances they might prove useful in diseases of copper excess.

The reader can get more information from these authors: Kuo et al., 2007 [6]; Harris 2001 [7]; Lutsenko et al. 2003 [8]; Moore et al. 2002 [9]. 


\section{Calcium/Cation/Ion Channels}

The majority of ion channels found in the cell membrane regulate the flow of metal ions. This includes sodium, potassium, and calcium in a variety of ligand and voltage activated configurations that can direct the flow of ions inward or outward. In general, calcium channels can be divided into high voltage types $(\mathrm{N}, \mathrm{P} / \mathrm{Q}, \mathrm{L}, \mathrm{R})$ and low voltage type $(\mathrm{T})$ each having their own electrophysiological and pharmacological characteristics. Calcium channels are also found in the sarcoplasmic reticulum regulating intracellular stores and $\mathrm{Ca}$ channels are also found in the mitochondrial membrane. Zinc and magnesium ions play important roles in regulating the conformational state of some receptors and channels; however, they are transported across the cell membrane by means other than ion channels and play no significant role in maintain resting or action potentials in the same way sodium and potassium do.

It would be surprising if oxidative stress did not effect the function of ion channels to some degree or another, yet this appears to be little investigated. Heavy metals, and other exogenous metals, exert their toxic effects through oxidative stress and it would seem logical that cellular structures that regulate ion flow or metal transport would likely be effected. Metals also exert some of their toxic effects by competing with physiologically essential cations, in particular calcium. Calcium L channels are blocked by $\mathrm{Sr}^{2+}$ and $\mathrm{Ba}^{2+}$. The trivalent cation of gadolinium is also an effective blocker of L-type calcium channels, although its action is different from the divalent cation blockers of $\mathrm{L}$ channels. There is a potential for other trivalent ions to alter the activity of calcium channels. Several heavy (e.g., $\mathrm{Hg}$ and $\mathrm{Pb}$ ) metals appear to block calcium channels on the cell membrane.

Toxic metals may alter calcium homeostasis by altering the conformational structure of the pumps, enzymes, binding proteins, and channels that regulate calcium flow. Often, this results in elevating free intracellular calcium levels which may produce depletion of glutathione/GSH with a downstream induction of DNA damage and eventual cell death. While ion channels play an important role in the response to metals and are no doubt effected by oxidative stress, the role of these proteins in regulating the response to oxidative stress in the brain is indirect. The pharmacology of the calcium channel has been extensively explored. However, manipulation of the calcium channel to prevent oxidative stress after exposure to metals has been little investigated and may have therapeutic potential. See Florea and Busselberg, 2009 [10]; Malasics et al., 2010 [12]; Simms and Zamponi, 2012 [11].

\section{Ceruloplasmin}

Ceruloplasmin $(\mathrm{Cp})$ is a large blood protein synthesized by the liver with the primary role of transporting copper. In the brain, $\mathrm{Cp}$ is bound to astroglia cell membrane or endoplasmic reticulum by glycosylphosphatidylinositol (GPI), although the exact function of the membrane bound form of $\mathrm{Cp}$ is unclear. Other blood proteins (albumin) also transport copper, but in smaller amounts. Ceruloplasmin is also a ferroxidase converting divalent iron to trivalent iron. If a disease process (e.g., hepatic failure) lowers the production of ceruloplasmin, the free copper would increase and copper mediated oxidative stress would be enhanced. Iron mediated oxidative stress would also increase because of the reduced rate of iron oxidation. Ceruloplasmin does play a specific role in the body's response to reactive oxygen species acting as a free-radical scavenger and possessing glutathione- (GSH-) peroxidase and nitric oxide- (NO-) oxidase/s-nitrosating activities. However, there is some evidence that under oxidative stress conditions, ceruloplasmin may induce further oxidative stress in a manner akin to a positive feedback mechanism. Also, when this protein is exposed to ROS, its ability to bind copper is reduced, releasing free copper, producing further oxidative stress. See Paradis et al., 2010 [13] and Shukla et al., 2006 [14].

\section{Catalase}

Catalase is a porphyrin heme containing protein that rapidly decomposes hydrogen peroxide to water and molecular oxygen. It is found in the greatest abundance in the peroxisome. Important to the role of metals in oxidative stress, many cationic metals can bind to the enzyme, inhibiting its activity. This may include both exogenously administered metals, but also metals freed from tissue damage. The removal of catalase from the redox cycle would indirectly increase oxidative stress which would be compounded by the presence of metals that may increase the production of ROS. The binding of metals to catalase appears to be covalent, limiting any therapeutic use of metals in altering the activity of this enzyme. Perhaps using catalase as a model for new drugs would be a better approach. This is reviewed extensively by Golden and Patel, 2009 [15].

\section{Copper Transporter 1}

Copper transporter 1 (Ctr1) has a high affinity for copper and serves to transport copper into the interior of the cell. Ctr1 is not the only mechanism by which this happens. DMT1 transports copper, but has a greater affinity for iron. Importantly, $\mathrm{Ctr} 1$ transports $\mathrm{Cu}^{1+}$, which has been converted from $\mathrm{Cu}^{2+}$ by a membrane bound reductase. It appears that Ctr1 transports $\mathrm{Cu}^{1+}$ into the cell when the ion binds to the high affinity site resulting in a conformational change and resulting pore that allows the $\mathrm{Cu}^{1+}$ to disengage from the molecule and move into the cell. The transport is not ATP dependent. As with many metal regulatory proteins, the structure of Ctr1 is highly conserved across species ranging from yeast to humans. It is expressed in high levels in the small intestine where it is responsible for transporting dietary copper into intestinal cells for further distribution to other tissues. It is also expressed in high levels in the liver and kidney. It is not as highly expressed in the brain, where the choroid plexus may contain the greater proportion. The lower levels of expression in the brain should not be taken as a sign that $\mathrm{Cu}$ metabolism is not important in the brain as several neural pathologies (Alzheimer's disease, spongiform 
encephalopathies) have been linked to disordered copper metabolism. The role of Ctr1 in copper metabolism has not been completely elucidated; in addition to transport into the cell, it also seems to play a role in the intracellular vesicular compartmentalization of the metal. Pharmacologic targets may include substances that bind to the high affinity site, prevent conformational changes, or block the pore formed by the Ctr1 molecule. All of these approaches would be useful in treating either disorders of copper excess or copper poisoning. For more information on this topic see Eisses and Kaplan, 2005 [16]; Lee et al., 2001 [17]; Nose et al., 2006 [18]; Sharp, 2003 [19].

\section{Copper Transport ATPase}

The copper transport ATPases7a and 7b, respective products of the so-called Menke's and Wilson's disease genes, are responsible for the transport of $\mathrm{Cu}$ across the cell membrane. Closely resembling each other, they have a key important difference, the direction in which they pump Cu. ATPase7a pumps $\mathrm{Cu}$ in an outward direction, removing $\mathrm{CU}$ from the interior. ATPase7b pumps $\mathrm{Cu}$ from the extracellular space to the interior.

ATPase7a has a large heavy metal binding domain containing six cysteine clusters. It is unclear what the function of this domain is. Both of the ATPases are found in the Golgi area of cells, where they appear to play a role in the movement of $\mathrm{Cu}$ into and out of internal cellular structures. Both ATPases are found in all tissues, but the liver contains a larger amount than other tissues. ATPase7a is strongly expressed in the choroid plexus.

The primary role of these two transporters is to play their part in keeping copper, a strong producer of free radicals, from doing damage. Pharmacological manipulation of these two proteins would include inhibition of function, although there would be little therapeutic values in such a maneuver. For in-depth reviews see Harris, 2000 [20]; La Fontaine and Mercer 2007 [21]; Prohaska and Gybina, 2004 [22]; Solioz et al., 1994 [23].

\section{Divalent Metal Transporter/NRAMP}

Divalent metal transporter 1 (DMT-1) (also known by the names divalent cation transporter 1 (DCT1) and natural resistance associated macrophage protein 2 (NRAMP2)) is found in all cells studied and is highly conserved across species. It is a fairly large protein of about $66 \mathrm{kDa}$ with 12 transmembrane domains. The genes are located on the human chromosome 12. Variations in expression have been reported.

DMT1 is capable of transporting a variety of divalent metals ( $\mathrm{Cd}, \mathrm{Co}, \mathrm{Cu}, \mathrm{Fe}, \mathrm{Mn}, \mathrm{Ni}, \mathrm{Pb}$, and $\mathrm{Zn}$ ) but its role in iron metabolism is the most closely examined. DMT1 transports ferrous iron into the cell by way of duodenal cytochrome B (dCytb) or endosomal bound transferrin, although the uptake of free iron has also been reported. The local and minor variations in subcelluar $\mathrm{pH}$ levels may be important in the manner in which iron is transported and DMT1 may also be a proton symporter. DMT1 also transports iron into the mitochondrial matrix. Changes in DMT1 levels appear to reflect changes in iron levels and the demand for iron. Other factors probably play a role in regulation of DMT1 levels and location in the cell, but they are poorly studied. DMT is found to some extent in all tissues, with the exact cellular location dependent on the space from which iron is absorbed. In the brain, it is found in neurons, glia, and capillary endothelial cells.

The role that DMT1 may play in disease is not well understood. During iron deficiency DMT1 is upregulated in the intestine and liver, but downregulated during iron loading. Mutations of DMT1 in the mk mouse and Belgrade rat produce hypochromic/microcytic anemia. Knockouts of the DMT1 gene produce animals that die of anemia.

DMT1 has been implicated in the transport of other metals including cadmium, copper, manganese, nickel, and zinc along with the neurodegenerative diseases associated with these metals. Iron accumulation is seen in the brain of patients with Alzheimer's, Parkinson's, and Huntington's diseases. However, evidence linking a dysfunction of DMT1 to these disorders is lacking. Excess iron accumulation after an ischemic event in the brain has been linked to regional oxidative stress. It has been suggested that pharmacologic blockade of DMT1 may be useful to prevent the uptake of iron in instances of ischemia. See Andrews, 2002 [24]; Mims and Prchal, 2005 [25]; Moos and Morgan, 2004 [26] for more detail.

\section{Ferritin}

Ferritin is a large globular protein involved in intracellular iron storage. As is typical of proteins involved in metal regulation, ferritin is highly conserved across species and found in all tissue types. Each ferritin molecule can store around 4500 iron atoms, in the ferric form. The intracellular version of the ferritin molecule is composed of heavy $(\mathrm{H})$ and light (L) subunits with the $\mathrm{H}$ subunit acting as an antioxidant ferroxidase while it acts to uptake iron. The $\mathrm{L}$ subunit plays a greater role in iron storage. The ratio of $\mathrm{H}$ to $\mathrm{L}$ subunits varies with the type of tissue and with a variety of stress and pathologic conditions. Inflammatory markers such as TNF-alpha, IL-1 beta, IL-6, and IL-1 alpha shift the ratio of heavy and light subunits in favor of the heavy subunits and thereby increase the amount of iron each ferritin molecule can store or they may simply increase the secretion of ferritin. It is unclear what the benefit of this might be. It may reduce the amount of iron available in fluid to form free radicals or may remove a potential iron source for invading microorganisms. The ratio of $\mathrm{H}$ to $\mathrm{L}$ may prove to be an important indicator of the type of stressor, or perhaps as an important biomarker. Serum ferritin has been studied as a marker for inflammatory processed, such as cardiovascular disease. Composed of L subunits, serum ferritin does correlate with a variety of other inflammatory markers and is elevated in inflammatory disease. However, its ultimate utility as a biomarker is clouded by its lack of specificity. 
Ferritin has been implicated in a number of pathologies. Ferritin has been shown to be upregulated in atherosclerotic vessels, suggesting a role in cerebral-vascular disease. It is unclear if the up-regulation is part of the atherosclerotic process proper, or if it is a protective mechanism induced by the atherosclerotic process. Ferritin synthesis is also induced by hypoxia and hypoxic reperfusion producing changes in ferritin levels that are complex. Both point to this molecule trying to mitigate free radical damage.

Interestingly, thyrotropin induces ferritin production. Thyrotropin deficiency (hypothyroidism) has been implicated in some instances of dementia-like disease, raising the possibility that abnormal iron metabolism may play a contributing role. Further discussion of this topic can be found in Balla et al., 1992 [27]; Cairo et al., 1995 [28]; Thomas et al., 1985 [29]; F. M. Torti and S. V. Torti, 2011 [30].

\section{Glutathione}

Glutathione (GSH) is a tripeptide consisting of cysteine, glycine, and glutamate. It is absolutely essential to the longterm health of a cell because of the role it plays as an antioxidant. Glutathione is synthesized from its component amino acids by gamma-glutamylcysteine synthetase and glutathione synthetase. The availability of cysteine is a determining factor in GSH synthesis and can limit the amount produced. GSH is conserved and recycled by the cell, once oxidized GSH forms glutathione disulfide (GSSH) which is converted back to GSH by glutathione reductase. The concentration of GSH in the cell is high, approaching $5 \mathrm{mM}$ in the liver. In addition to acting as an antioxidant, GSH also plays a role in a variety of cellular reactions such as DNA repair and enzyme activation. In the brain, GSH production, metabolism, excretion, and synthesis are large and energetically important. GSH and GSSH secretion may play a role in the functioning of a variety of receptors. Interestingly, neurons and astrocytes appear to engage in a metabolic exchange of GSH and its precursors implying a codependence between these two cell types for an optimal response to oxidative stress.

GSH depletion has been implicated in nearly every disease state of the brain and probably indicates that oxidative stress plays a broad role in many diseases and certainly suggests that some disorder of GSH metabolism may be responsible for some neuropathologies. This certainly appears to be the case for Parkinson's disease where evidence points both to a defect in GSH synthesis and increased oxidative stress and an increased oxidative stress response in the substantia nigra. Dopaminergic neurons may be especially sensitive to oxidative stress because of the oxidative species generated by dopamine synthesis. Additionally, GSH depletion may interfere with mitochondrial function, leading to further neural stress. Further complicating the picture are several studies by Muller and coworkers [31-33] suggesting that the administration of levodopa, one of the primary means of treating PD, may increase oxidative stress by producing ROS and depleting cysteine levels. Further work along this line needs to be done for any number of therapeutic agents. Supplementation with GSH precursors has proven useful in some neuropathological conditions. More can be found on this topic in Dringen et al., 2000 [34]; Dringen and Hirrlinger, 2003 [35]; Martin and Teismann, 2009 [36]; Schulz et al., 2000 [37].

\section{Hepcidin}

In the broadest sense, hepcidin is an iron regulatory hormone secreted by the liver that inhibits iron absorption from the small intestine. Hepcidin additionally inhibits the release of iron from macrophages and the transport of iron across the placenta. Hepcidin secretion is reduced in simple iron deficiency, such as an iron poor diet, and hypoxia presumably to allow more iron to be absorbed and allow for increased erythropoiesis. Erythropoietin has been shown to inhibit hepcidin synthesis. Conversely, iron overload increases the secretion of hepcidin. Hepcidin appears to bind to the exporter ferroportin channel of enterocytes and macrophages inhibiting the secretion of iron and retaining iron in those cells. Other cell types may also be affected. The exact cellular mechanism by which hepcidin secretion is triggered or inhibited is unknown and requires exploration.

Hepcidin is synthesized initially as an 84 amino acid preprohormone and undergoes conversion to 60 then 39 amino acid forms and then ultimately to the 25 amino acid form active in circulation. It is excreted in urine as 20 and 22 amino acid degradation forms at the same rate it is produced in the body. The structure of hepcidin contains beta sheets and a hairpin structure with 4 disulfide bonds. The structure of hepcidin is well conserved across mammalian species.

The role of hepcidin in iron regulation gives it a unique role in inflammation and infection. Lipopolysaccharide and the inflammatory cytokine interleukin-6 induce the production of hepcidin. The types of cytokines that induce the production of hepcidin, and those that do not, suggest that it is a type II acute phase inflammatory protein. The resultant sequestration of iron in macrophages is believed to be a strategy to deprive invading microorganisms of iron. In this regard hepcidin was initially considered an antimicrobial peptide.

The over secretion of hepcidin is a major factor in the anemia of inflammation, the result of a variety of disease processes. Conversely, low secretion of hepcidin is associated with conditions where iron overload occurs (e.g., betathalassemia and hemochromatosis).

Pharmacologic manipulation of hepcidin will be difficult until the exact cellular regulatory mechanism is elucidated. Certainly it would be theoretically possible to administer hepcidin in instances where a deficient secretion is responsible for iron overload. A more fruitful approach would be to administer some types of ligands that would promote the secretion. Inhibition of secretion in disease processes associated with the anemia of inflammation would require the administration of a compound that would inhibit the secretion of hepcidin from the liver. More on hepcidin can be found in the articles by Kemna et al, 2008 [38]; Nemeth et al., 2003 [39]; Ganz, 2003 [40]. 


\section{Matrix Metalloproteinases}

Matrix metalloproteins (MMPs) are zinc containing proteases involved in a variety of cell functions ranging from cell division to cell death and cell adhesion. There are nearly 30 different MMPs that have been identified and all share common structural and functional features. A wide variety of MMPs are expressed in all tissues with no single MMP being a strict marker for a specific tissue. However, there does seem to some preferential expression of particular MMPs in various tissues.

MMPs are of particular interest because of their ability to degrade receptors and the extracellular matrix. MMPs are activated with tissue injury or inflammation where they are involved in remodeling of the extracellular matrix for tissue repair. Under physiologically ideal outcomes, MPPs remodel the extracellular matrix to reflect a healed state and promote the death of injured or dying cells but do not stimulate apoptosis of healthy cells. In general, MMPs promote cell proliferation and survival.

The activity of MMPs is regulated by a variety of mechanisms including tissue inhibitors of metalloproteinases (TIMPs), of which there are four types. MMPs are secreted by the cell, in response to a variety of stimuli, and then activated when the so-called cysteine switch is cleaved by any number of enzyme systems. MMPs are of particular interest in diseases that involve cell proliferation or migration, cancer being an important pathology where MMPs play a key role in promoting abnormal cell proliferations, tissue invasion, and angiogenesis. In the CNS, the role of MMPs in neural pathology is less obvious, except in the case of CNS tumors. In the CNS, MMPs 1, 2, 3, and 9 are more common than the other types. It also appears that microglia and mononuclear phagocytes are proportionately more active producers of MMPs, although MMP-9 is more dominant in neurons.

Exposure of neurons, and other cells, to oxidative stress does not seem to directly alter MMP activity. Rather, a variety of events leads to some change in the production of MMPs or TIMPs. The "typical" response appears to be an increase in MMP as a part of the inflammatory process, resulting in tissue remodeling, both beneficial (wound repair) and destructive (metastasis). Oxidative stress is correlated with the production of MPPs, but is not the direct signal for MPP upregulation.

There is some evidence that MMPs may play a role in Alzheimer's disease AD, AIDS related dementia, and any other neuropathology where inflammation plays a role. The increase in ROS seen in AD seem to be linked, although indirectly, to an increase in MMP 2 and MMP 9. This may be associated with a breakdown in the blood brain barrier further compromising brain homeostasis on a number of levels. The role of MMPs in HIV-related dementias is linked to its role in the inflammatory cascade.

Pharmacologic targets related to the MMPs would be related to the inflammatory process that alters MMP activity, the direct inhibition of MMP, or the activation of TIMP. However, these approaches are fraught with problems because of the diverse roles that MMPs and their regulators play in tissue structure and function. Please refer to the following reviews for more detail on this topic: Amălinei et al., 2010 [41]; Bell and Zlokovic, 2009 [42]; Chao and Ghorpade, 2009 [43]; Michaluk and Kaczmarek, 2007 [44].

\section{Metallothionein}

Metallothionein (MT) is a cysteine rich protein involved in the regulation of zinc and other metals. This protein is found in a variety of forms, depending on the species in question. In mammals, MT forms I, II, III, and IV are found in a variety of tissues. MT I and II are the most abundant in the CNS. In the CNS, MT is found mostly in astrocytes. MT I and II are also found in ependymal cells, pia mater, and endothelial cells of cerebral blood vessels. MT III is specific to the brain but is not abundantly expressed. MT III also exhibits several characteristics that make it different from the more dominant forms of MT, MT I, and II. Unless otherwise noted, when speaking of MT in this context, we will be discussing MT I and II.

MT binds up to 7 divalent and up to 12 monovalent cations. In terms of normal physiology, these are typically zinc, copper, and selenium. However, a variety of metals important to toxicologists and pharmacologists are also bound and these include arsenic, cadmium, mercury, uranium, and other heavy and transitional metals.

In addition to sequestering heavy metals, MT plays an important role in cell signaling. This involves the binding of zinc ions and releasing them in response to physiologic cues, which in the CNS includes synaptic signaling at zinc containing synapses. MT is also involved in the regulation of transcription factors that involve zinc finger proteins.

MT expression is inducible in response to oxidative stress and a variety of other stimuli including metals, glucocorticoids, thyroid hormone, and inflammatory cytokines.

MT is involved in the response to oxidative stress. Free radicals, such as the superoxide and hydroxyl radical, oxidize the cysteine residue quelling their potential for other cellular damage. In turn, the bound metal is released into the cellular fluid. If that metal is zinc this may stimulate the further production of MT allowing for further control of oxidative stress. Metal induced synthesis is further promoted by the metal responsive element (MRE) found in the promoter region of the $M T$ gene.

MT expression is also induced in a variety of tissue injuries, presumably via the inflammatory response. In the brain, this includes head injury and cerebral vascular accidents. The expression of MT after CNS damage is complex. The simplest response may be an initial downregulation followed by an upregulation. MT III has been associated with Alzheimer's disease. Given MT's central role in cellular physiology and its association with inflammation one would be hard-pressed to find a disease process where MT is not involved.

Pharmacologic targets of MT are varied. In many ways, a variety of agents that target MT are already available. These would include glucocorticoids that target the glucocorticoid responsive elements (GREs) of the MT gene. Similarly, thyroid hormone (thyroid response element: TRE) induces MT. 
Cytokines are available for administration (e.g., interleukins) and induce MT production by way of the signal transducer and activator of transcription (STAT) factors. We must not forget that metals themselves alter MT expression and function. It is reasonable that metal administration could be tailored to take advantage of their MT inducing properties and alter its activity in a desirable fashion. There is of course the possibility of the direct administration of MT. In the CNS the administration method would need to circumvent the blood brain barrier.

Experimental results with mice modified to over- or underexpress MT suggest that pharmacologic approaches that would up-regulate the expression of MT would be useful in the treatment of cerebral vascular accidents and head injury. The use of MT and MT active drugs in spinal cord and brain injury is particularly intriguing because MT promotes neuronal sprouting. There may be a similar benefit for multiple sclerosis. Whether or not the pharmacologic manipulation of MT in neurodegenerative disease such as Alzheimer's disease would be beneficial is uncertain. MT expression is elevated in $\mathrm{AD}$ but it is unclear if this is in response to the disease process or part of the disease process. This is additionally confounded because one form of MT (MT III) may be acting as an inhibitory growth factor for synapses. This needs to be determined experimentally in the appropriate animal model. Lastly, there is the theoretical possibility for the pharmacologic manipulation of MT in instances of metal poisoning (see [45]). See West et al., 2008 [46] for a complete review.

\section{Superoxide Dismutase}

Superoxide dismutase (SOD) is a metalloenzyme that transforms the superoxide into less dangerous oxygen and hydrogen peroxide. It is ubiquitous and found in nearly all life forms. It is evolutionarily well preserved. In humans and mammals it is found in three basic forms. SOD1 (also CuZnSOD) is found in intracellular cytoplasm. It is a dimer with a weight of 32000 and uses a copper and zinc atom as part of its structure. SOD2 (also MnSOD) is localized in mitochondria and uses manganese as its functional metal. It is a tetramer with a weight of 88000 . SOD3 (sometimes ecSOD) is located in extracellular fluid and also uses copper and zinc as its metal component. It is a tetramer and the heaviest of the three with a weight of 120000 . Found in all tissues, SOD is found in particularly high concentrations in the liver, which is used as an industrial source of the enzyme.

At one level or another, SOD can be implicated in any disease in which oxidative stress is a factor. However, a specific deficit in SOD1 dysfunction has been implicated in amyotrophic lateral sclerosis and mnSOD has been implicated in some polyneuropathies. All of the SOD isoforms have been implicated in ischemic damage, in particular that they are exhausted in the ischemic hypoxic state. When perfusion is reestablished, free radicals are produced faster than new SOD can be synthesized leading to greater tissue damage. Therapeutic strategies have been focused on reintroducing SOD into the ischemic area. This is particularly challenging because of SODs large size, which is particularly problematic when it comes to crossing the blood brain barrier. A variety of approaches are being tried to get SOD across the BBB including liposomes and conjugation with a variety of transport ligands. For more reviews see Chan, 2001 [47]; Maritim et al., 2003 [48]; Tsay et al., 2000 [49]; Xiong et al., 2005 [50].

\section{Conclusion}

The number of proteins involved in metal oxidative stress is large but not bewildering. In the broadest sense, these proteins fall into two groups: those involved in iron or copper metabolism, and those involved with the rest of the metals. Iron and copper have such significant redox activity that a number of regulatory mechanisms have evolved specifically for the control of these metals. When the mechanisms for controlling the redox activity of these metals fail, then some disease process eventually develops. Metals other than iron and copper may produce oxidative stress through mechanisms that often, but not always, involve binding to a cysteine molecule. Pharmacologic targets are abundant in all these systems but the challenge will be to develop ligands that improve the functioning of the system, but without simply shifting the oxidative stress to another set of molecules.

\section{References}

[1] K. Oettl and R. E. Stauber, "Physiological and pathological changes in the redox state of human serum albumin critically influence its binding properties," British Journal of Pharmacology, vol. 151, no. 5, pp. 580-590, 2007.

[2] L. Turell, S. Carballal, H. Botti, R. Radi, and B. Alvarez, "Oxidation of the albumin thiol to sulfenic acid and its implications in the intravascular compartment," Brazilian Journal of Medical and Biological Research, vol. 42, no. 4, pp. 305-311, 2009.

[3] A. E. Finefrock, A. I. Bush, and P. M. Doraiswamy, "Current status of metals as therapeutic targets in Alzheimer's disease," Journal of the American Geriatrics Society, vol. 51, no. 8, pp. 1143-1148, 2003.

[4] C. J. Maynard, A. I. Bush, C. L. Masters, R. Cappai, and Q.-X. Li, "Metals and amyloid- $\beta$ in Alzheimer's disease," International Journal of Experimental Pathology, vol. 86, no. 3, pp. 147-159, 2005.

[5] H. Zheng and E. H. Koo, "Biology and pathophysiology of the amyloid precursor protein," Molecular Neurodegeneration, vol. 6, no. 1, article 27, 2011.

[6] M. T. Kuo, H. H. W. Chen, I. S. Song, N. Savaraj, and T. Ishikawa, "The roles of copper transporters in cisplatin resistance," Cancer and Metastasis Reviews, vol. 26, no. 1, pp. 71-83, 2007.

[7] E. D. Harris, "Copper homeostasis: the role of cellular transporters," Nutrition Reviews, vol. 59, no. 9, pp. 281-285, 2001.

[8] S. Lutsenko, R. Tsivkovskii, and J. M. Walker, "Functional properties of the human copper-transporting ATPase ATP7B (the Wilson's disease protein) and regulation by metallochaperone Atox1," Annals of the New York Academy of Sciences, vol. 986, pp. 204-211, 2003.

[9] S. D. P. Moore, K. E. Helmle, L. M. Prat, and D. W. Cox, "Tissue localization of the copper chaperone ATOX1 and its 
potential role in disease," Mammalian Genome, vol. 13, no. 10, pp. 563-568, 2002.

[10] A.-M. Florea and D. Büsselberg, "Anti-cancer drugs interfere with intracellular calcium signaling," NeuroToxicology, vol. 30, no. 5, pp. 803-810, 2009.

[11] B. A. Simms and G. W. Zamponi, "Trafficking and stability of voltage-gated calcium channels," Cellular and Molecular Life Sciences, vol. 69, no. 6, pp. 843-856, 2012.

[12] A. Malasics, D. Boda, M. Valiskó, D. Henderson, and D. Gillespie, "Simulations of calcium channel block by trivalent cations: $\mathrm{Gd}^{3+}$ competes with permeant ions for the selectivity filter," Biochimica et Biophysica Acta, vol. 1798, no. 11, pp. 2013-2021, 2010.

[13] M. Paradis, J. Gagné, M. A. Mateescu, and J. Paquin, "The effects of nitric oxide-oxidase and putative glutathioneperoxidase activities of ceruloplasmin on the viability of cardiomyocytes exposed to hydrogen peroxide," Free Radical Biology and Medicine, vol. 49, no. 12, pp. 2019-2027, 2010.

[14] N. Shukla, J. Maher, J. Masters, G. D. Angelini, and J. Y. Jeremy, "Does oxidative stress change ceruloplasmin from a protective to a vasculopathic factor?" Atherosclerosis, vol. 187, no. 2, pp. 238-250, 2006.

[15] T. R. Golden and M. Patel, "Catalytic antioxidants and neurodegeneration," Antioxidants and Redox Signaling, vol. 11, no. 3, pp. 555-569, 2009.

[16] J. F. Eisses and J. H. Kaplan, "The mechanism of copper uptake mediated by human CTR1: a mutational analysis," Journal of Biological Chemistry, vol. 280, no. 44, pp. 37159-37168, 2005.

[17] J. Lee, J. R. Prohaska, and D. J. Thiele, "Essential role for mammalian copper transporter Ctr1 in copper homeostasis and embryonic development," Proceedings of the National Academy of Sciences of the United States of America, vol. 98, no. 12, pp. 6842-6847, 2001.

[18] Y. Nose, B. E. Kim, and D. J. Thiele, "Ctr1 drives intestinal copper absorption and is essential for growth, iron metabolism, and neonatal cardiac function," Cell Metabolism, vol. 4, no. 3, pp. 235-244, 2006.

[19] P. A. Sharp, "Ctr1 and its role in body copper homeostasis," International Journal of Biochemistry and Cell Biology, vol. 35, no. 3, pp. 288-291, 2003.

[20] E. D. Harris, "Cellular copper transport and metabolism," Annual Review of Nutrition, vol. 20, pp. 291-310, 2000.

[21] S. La Fontaine and J. F. B. Mercer, "Trafficking of the copperATPases, ATP7A and ATP7B: role in copper homeostasis," Archives of Biochemistry and Biophysics, vol. 463, no. 2, pp. 149-167, 2007.

[22] J. R. Prohaska and A. A. Gybina, "Intracellular copper transport in mammals," Journal of Nutrition, vol. 134, no. 5, pp. 1003-1006, 2004.

[23] M. Solioz, "Copper pumping ATPases: common concepts in bacteria and man," FEBS Letters, vol. 346, no. 1, pp. 44-47, 1994.

[24] N. C. Andrews, "Metal transporters and disease," Current Opinion in Chemical Biology, vol. 6, no. 2, pp. 181-186, 2002.

[25] M. P. Mims and J. T. Prchal, "Divalent metal transporter 1," Hematology, vol. 10, no. 4, pp. 339-345, 2005.

[26] T. Moos and E. H. Morgan, "The metabolism of neuronal iron and its pathogenic role in neurological disease review," Annals of the New York Academy of Sciences, vol. 1012, pp. 14-26, 2004.

[27] G. Balla, H. S. Jacob, J. Balla et al., "Ferritin: a cytoprotective antioxidant strategem of endothelium," Journal of Biological Chemistry, vol. 267, no. 25, pp. 18148-18153, 1992.

[28] G. Cairo, L. Tacchini, G. Pogliaghi, E. Anzon, A. Tomasi, and A. Bernelli-Zazzera, "Induction of ferritin synthesis by oxidative stress. Transcriptional and post-transcriptional regulation by expansion of the "free" iron pool," Journal of Biological Chemistry, vol. 270, no. 2, pp. 700-703, 1995.

[29] C. E. Thomas, L. A. Morehouse, and S. D. Aust, "Ferritin and superoxide-dependent lipid peroxidation," The Journal of Biological Chemistry, vol. 260, no. 6, pp. 3275-3280, 1985.

[30] F. M. Torti and S. V. Torti, "Regulation of ferritin genes and protein," Blood, vol. 99, no. 10, pp. 3505-3516, 2002.

[31] T. Müller and W. Kuhn, "Homocysteine levels after acute levodopa intake in patients with Parkinson's disease," Movement Disorders, vol. 24, no. 9, pp. 1339-1343, 2009.

[32] T. Müller and S. Muhlack, "Cysteinyl-glycine reduction as marker for levodopa-induced oxidative stress in Parkinson's disease patients," Movement Disorders, vol. 26, no. 3, pp. 543546, 2011.

[33] T. Muller and S. Muhlack, "Cysteine decrease following acute levodopa intake in patients with Parkinson's disease," Neuroscience Letters, vol. 521, pp. 37-39, 2012.

[34] R. Dringen, J. M. Gutterer, and J. Hirrlinger, "Glutathione metabolism in brain: metabolic interaction between astrocytes and neurons in the defense against reactive oxygen species," European Journal of Biochemistry, vol. 267, no. 16, pp. 49124916, 2000.

[35] R. Dringen and J. Hirrlinger, "Glutathione pathways in the brain,” Biological Chemistry, vol. 384, no. 4, pp. 505-516, 2003.

[36] H. L. Martin and P. Teismann, "Glutathione-a review on its role and significance in Parkinson's disease," FASEB Journal, vol. 23, no. 10, pp. 3263-3272, 2009.

[37] J. B. Schulz, J. Lindenau, J. Seyfried, and J. Dichgans, "Glutathione, oxidative stress and neurodegeneration," European Journal of Biochemistry, vol. 267, no. 16, pp. 4904-4911, 2000.

[38] E. H. J. M. Kemna, H. Tjalsma, H. L. Willems, and D. W. Swinkels, "Hepcidin: from discovery to differential diagnosis," Haematologica, vol. 93, no. 1, pp. 90-97, 2008.

[39] E. Nemeth, E. V. Valore, M. Territo, G. Schiller, A. Lichtenstein, and T. Ganz, "Hepcidin, a putative mediator of anemia of inflammation, is a type II acute-phase protein," Blood, vol. 101, no. 7, pp. 2461-2463, 2003.

[40] T. Ganz, "Hepcidin, a key regulator of iron metabolism and mediator of anemia of inflammation," Blood, vol. 102, no. 3, pp. 783-788, 2003.

[41] C. Amălinei, D. Căruntu I, S. E. Giușcă, and R. A. Bălan, "Matrix metalloproteinases involvement in pathologic conditions," Romanian Journal of Morphology and Embryology, vol. 51, no. 2, pp. 215-228, 2010.

[42] R. D. Bell and B. V. Zlokovic, "Neurovascular mechanisms and blood-brain barrier disorder in Alzheimer's disease," Acta Neuropathologica, vol. 118, no. 1, pp. 103-113, 2009.

[43] C. Chao and A. Ghorpade, "Production and roles of glial tissue inhibitor of metalloproteinases-1 in human immunodeficiency virus-1-associated dementia neuroinflammation: a review," American Journal of Infectious Diseases, vol. 5, no. 4, pp. 314-320, 2009.

[44] P. Michaluk and L. Kaczmarek, "Matrix metalloproteinase-9 in glutamate-dependent adult brain function and dysfunction," Cell Death and Differentiation, vol. 14, no. 7, pp. 1255-1258, 2007.

[45] Y. Hao, J. Ren, J. Liu et al., "The protective role of zinc against acute toxicity of depleted uranium in rats," Basic \& Clinical Pharmacology \& Toxicology, vol. 111, no. 6, pp. 402-410, 2012.

[46] A. K. West, J. Hidalgo, D. Eddins, E. D. Levin, and M. Aschner, "Metallothionein in the central nervous system: roles in protection, regeneration and cognition," Neurotoxicology, vol. 29, no. 3, pp. 489-503, 2008. 
[47] P. H. Chan, "Reactive oxygen radicals in signaling and damage in the ischemic brain," Journal of Cerebral Blood Flow and Metabolism, vol. 21, no. 1, pp. 2-14, 2001.

[48] A. C. Maritim, R. A. Sanders, and J. B. Watkins, "Diabetes, oxidative stress, and antioxidants: a review," Journal of Biochemical and Molecular Toxicology, vol. 17, no. 1, pp. 24-38, 2003.

[49] H.-J. Tsay, P. Wang, S.-L. Wang, and H.-H. Ku, "Age-associated changes of superoxide dismutase and catalase activities in the rat brain," Journal of Biomedical Science, vol. 7, no. 6, pp. 466474, 2000.

[50] Y. Xiong, F. S. Shie, J. Zhang, C. P. Lee, and Y. S. Ho, "Prevention of mitochondrial dysfunction in post-traumatic mouse brain by superoxide dismutase," Journal of Neurochemistry, vol. 95, no. 3, pp. 732-744, 2005. 


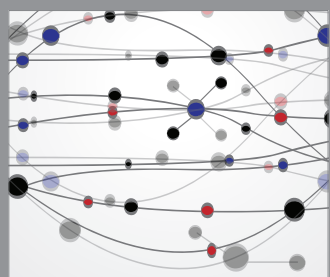

The Scientific World Journal
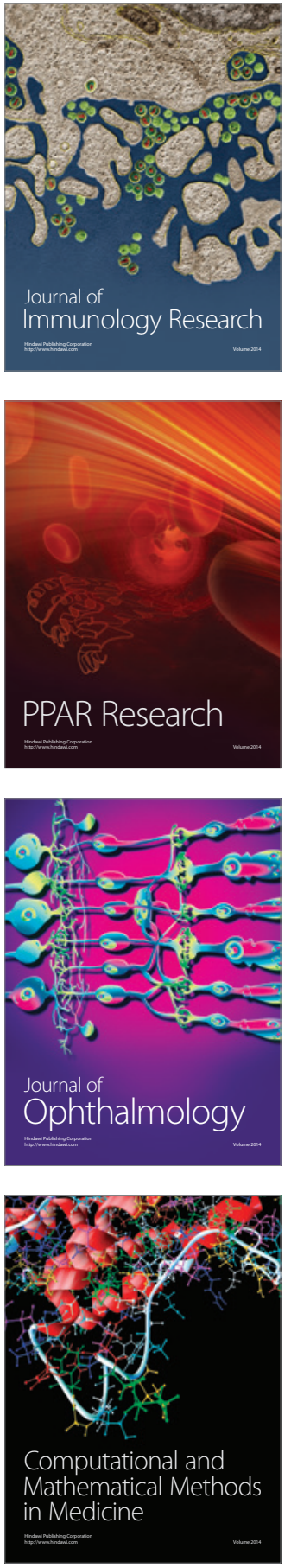

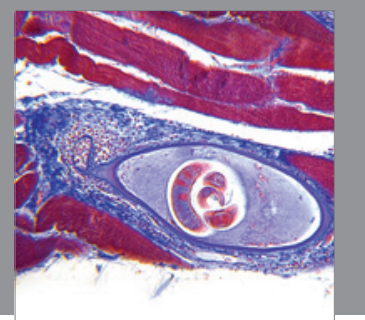

Gastroenterology

Research and Practice
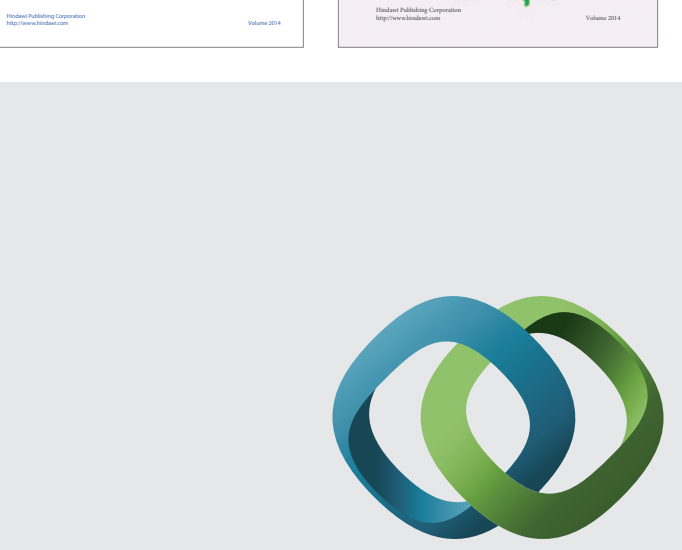

\section{Hindawi}

Submit your manuscripts at

http://www.hindawi.com
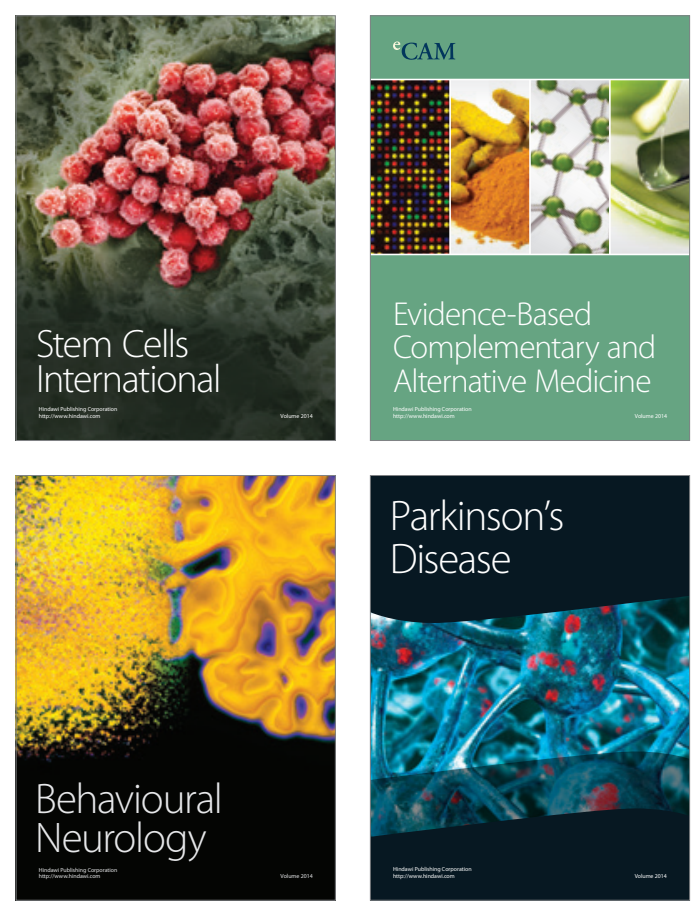

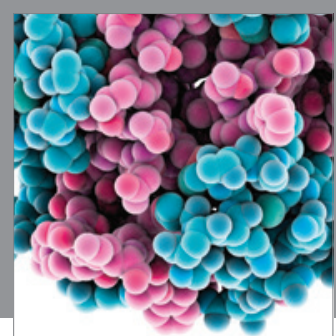

Journal of
Diabetes Research

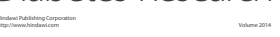

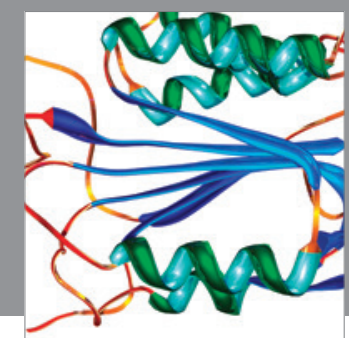

Disease Markers
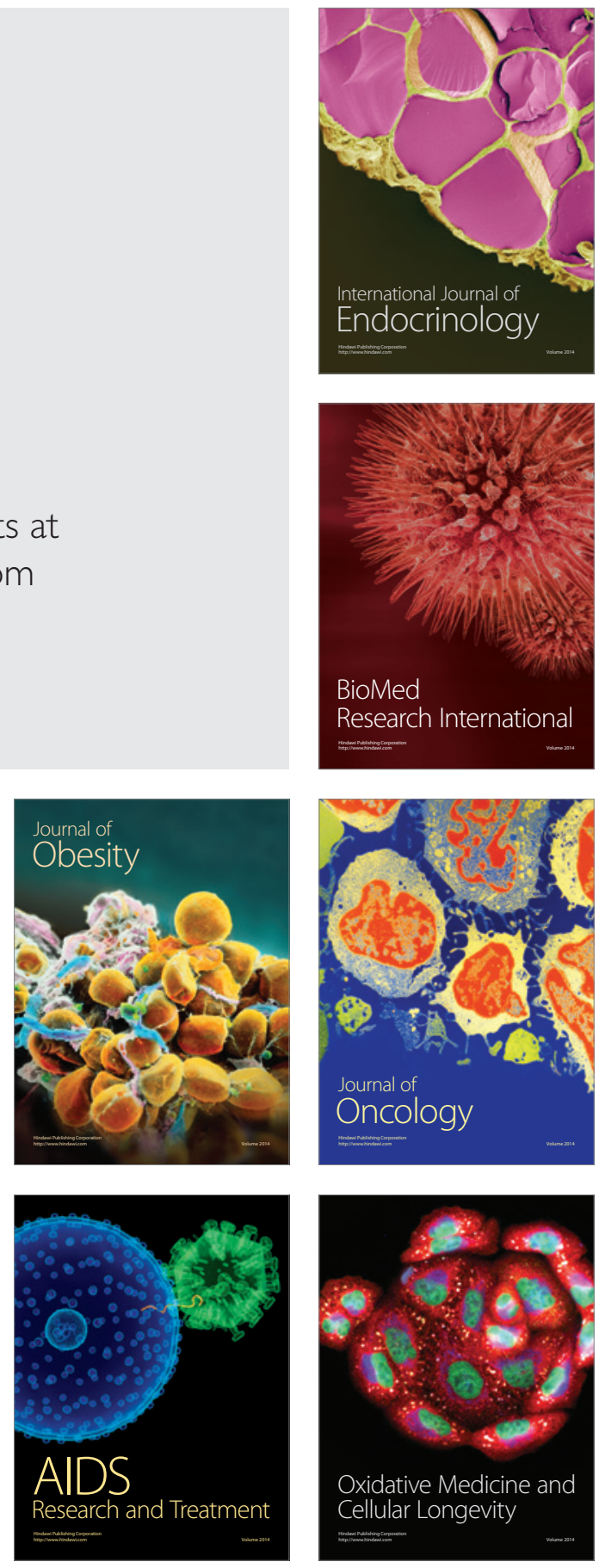